\title{
CRECIMIENTO EMPRESARIAL: ESTRATEGIA DE DESARROLLO DEL MERCADO EN EL SECTOR MIPYMES
}

\author{
BUSINESS GROWTH: MARKET DEVELOPMENT STRATEGY IN \\ THE MIPYMES SECTOR
}

\author{
Xiomara Nayel Yoza Calderón ${ }^{1}$, Ronny Anthony Villafuerte Soledispa ${ }^{2}$, María Leono Parrales \\ Poveda $^{3}$
}

\section{1,2,3 - Universidad Estatal del Sur de Manabí, Manabí, Ecuador}

\begin{abstract}
1. Email: yoza-xiomara1642@unesum.edu.ec ORCID: https://orcid.org/0000-0003-0535-8610
\end{abstract}
2. Email: villafuerte-ronny2040@unesum.edu.ec ORCID: https://orcid.org/0000-0003-2002-7836

3. Email: maria.parrales@unesum.edu.ec ORCID: https://orcid.org/0000-0003-3994-3711

Recibido: 11/05/2021 Aceptado: 20/07/2021

Para Citar: Yoza Calderón, X. N., Villafuerte Soledispa, R. A., \& Parrales Poveda, M. L. (2021). Crecimiento empresarial: estrategia de desarrollo del mercado en el sector MIPYMES. Revista Publicando, 8(31), 82-95. https://doi.org/10.51528/rp.vol8.id2236

\begin{abstract}
Resumen:
Actualmente, el sector de las Mipymes se encuentra en medio de condiciones adversas, caracterizadas por la sobreoferta de productos y servicios y altos niveles de incertidumbre; estas y otras manifestaciones externas las han llevado a dedicar gran parte del esfuerzo administrativo, a la búsqueda de nuevas alternativas que permitan contrarrestar los efectos de la competencia y los drásticos cambios comerciales a nivel mundial. El presente estudio se enfoca en analizar las estrategias de desarrollo del mercado que han permitido que muchas Mipymes ecuatorianas logren un crecimiento empresarial y sobre todo la sostenibilidad en el mercado. A través de una investigación de tipo descriptiva, bajo un enfoque cualitativo, no experimental se caracterizaron de manera crítica las estrategias de desarrollo de mercados que presentan las Mipymes en el Ecuador. Los resultados muestran que la creatividad e innovación y la resiliencia con la que operan los negocios han sido clave para mantenerse en el mercado.
\end{abstract}

Palabras clave: desarrollo empresarial, estrategias, ventaja competitiva.

\begin{abstract}
:
Currently, the MSME sector is in the midst of adverse conditions, characterized by the oversupply of products and services and high levels of uncertainty; these and other external manifestations have led them to devote much of the administrative effort to the search for new alternatives to counteract the effects of competition and drastic commercial changes worldwide. This study focuses on analyzing the market development strategies that have allowed many Ecuadorian MSMEs to achieve business growth and, above all, sustainability in the market. Through a descriptive type of research, under a qualitative, non-experimental approach, the market development strategies presented by MSMEs in Ecuador were critically characterized. The results show that creativity and innovation and the resilience with which the businesses operate have been key to remain in the market.
\end{abstract}

Keywords: business development, competitive advantage, strategies. 


\section{INTRODUCCIÓN}

as micro, pequeñas y medianas empresas
(Mipymes) hoy en día, debido al
fortalecimiento tecnológico y a la
constante entrada de nuevos competidores, deben buscar diferenciarse en su producción y orientarse a la apertura de nuevos mercados. Estos mercados inciertos requieren de la ubicación e identificación de la demanda, lo que obliga a la empresa a adoptar estrategias de desarrollo en los productos ajustados a las necesidades del usuario y del mercado y a las posibilidades tecnológicas y productivas de la organización.

El crecimiento empresarial y sus estrategias de desarrollo del mercado son factores cada vez más importantes dentro de los procesos de producción, se convierten en ese conjunto de acciones que conducen a la consecución de una ventaja competitiva sostenible en el tiempo y factible de ser defendida ante la competencia con el fin de satisfacer los objetivos y necesidades de los diversos grupos participantes en el mercado.

Según Adame et al., (2017) en su trabajo de investigación Crecimiento empresarial \& Mipymes de Autlán de Navarro de la Revista Cubana de Contabilidad y Finanzas, describe que las microempresas se desarrollan a partir de un proyecto emprendedor por parte del dueño, el cual tiene la cualidad de ser al mismo tiempo administrador de su propia empresa. Estos negocios venden sus productos a baja escala y no requieren de gran inversión para su funcionamiento, por lo que tienen la posibilidad de crecer y generar competitividad con sus similares. La importancia de estas empresas radica en su contribución al desarrollo local, a la creación de empleos y al aumento del ingreso familiar, donde se aprovechan los recursos internos que existen en las áreas rurales y en los centros urbanos.

Este estudio tiene como objetivo analizar las estrategias orientadas a conseguir posicionarse mejor en el nicho escogido, con el producto creado y del que se espera aporte a ese anhelado desarrollo empresarial.

En ese sentido, para lograr apoderarse de la mente del comprador, la empresa debe seguir ciertos lineamientos estratégicos, uno de ellos se enfoca en saber comunicar a su personal cuáles son las necesidades del cliente y cómo satisfacerlas. Parrales et al. (2017) en su libro Lineamientos estratégicos y comunicación digital enfatiza que:

Saber comunicar puede contribuir a incrementar las competencias de las empresas e instituciones, porque éstas, constantemente, se retroalimentan de las críticas y sugerencias de los empleados, clientes, proveedores, entre otros, quienes, al ser atendidos por el nivel ejecutivo, sin restricciones, se sienten motivados y comprometidos con su evolución, a través del sentido de pertenencia y de un clima de trabajo integrador. Posicionar la marca de una empresa o institución entre los consumidores, puede lograrse mediante una estrategia que incluya una buena comunicación. Esta es una herramienta vital que se debe utilizar en el marco de ciertos lineamientos estratégicos, que tengan en cuenta la imagen que en el mercado se quiera lograr posicionar. (p. 10)

Desde la perspectiva de Macías (2015), las Mipymes cumplen un rol fundamental para el 
desarrollo de la economía, pues la producción de bienes y servicios satisface una demanda existente, lo que constituye un eslabón determinante en el encadenamiento de la actividad económica y generación de empleo, son asociaciones solidarias y su trascendencia radica en que asegura el mercado laboral por medio de la descentralización de la mano de obra, posibilita la concentración de la renta y la capacidad provechosa a partir de un número limitado de organizaciones hacia uno más grande, muestran mayor adaptabilidad tecnológica y menor precio de infraestructura, son unidades parientes que permiten una interacción más estrecha entre el empleador y el empleado.

Actualmente, las empresas se encuentran en medio de condiciones adversas, caracterizadas por la sobreoferta de productos y servicios y altos niveles de incertidumbre; estas y otras manifestaciones externas las han llevado a dedicar gran parte del esfuerzo administrativo a la búsqueda de nuevas alternativas que permitan contrarrestar los efectos de la competencia y los drásticos cambios comerciales a nivel mundial. En este panorama, las empresas intentan desarrollar e implementar políticas y estrategias que les permitan crecer y consolidarse (Aguilera y Puerto 2012).

Cancino et al. (2012) mencionan que la actividad emprendedora fue considerada desde siempre un mecanismo fundamental para el desarrollo económico, este vínculo se observa cuando los emprendedores inventan nuevos negocios, los nuevos negocios necesitan producir empleos, se intensifica la competencia e inclusive se incrementa la productividad por medio de cambios tecnológicos.
El mercado competitivo y saturado en que conviven las Mipymes les obliga a desarrollar estrategias de mercado para alcanzar el crecimiento empresarial, las mismas consisten en identificar y desarrollar nuevos segmentos de mercado para productos existentes, así mismo se define como una serie de planeamientos destinados a ampliar el nicho de una determinada empresa y por ende, de hacerlo más grande y potencialmente más rentable para el desarrollo de una actividad económica concreta (Riquelme, 2015).

El desarrollo de las Mipymes y el impacto al aplicar estrategias de desarrollo de mercado, debe ser una prioridad para su crecimiento económico, ¿cómo se logra?, creando valor y detectando nuevas necesidades y deseos en el mercado. Si las Mipymes quieren mantenerse, crecer $y$ desarrollarse en un entorno más amplio y dinámico deben plantear estrategias que les permitan alcanzar su desarrollo empresarial. Si una empresa quiere perdurar competitivamente en el mercado debe plantearse de forma constante el desarrollo de estrategias de crecimiento, pero no sólo para mejorar las ventas, la cuota de mercado, el beneficio o el tamaño de la organización, sino también para sobrevivir a los ataques de la competencia (Ceupe, 2016).

\section{Fuerzas competitivas que moldean las estrategias de las Mipymes}

Según Porter (citado por Donawa, 2018) las cinco fuerzas que moldean la estrategia de las empresas han sido analizadas por varios autores como Koontz, Weihrich y Cannice. Explican que Porter sugiere que la formulación de estrategias 
requiere un análisis del atractivo de una industria y la posición de la compañía dentro de ella y este análisis se convierte en la base para formular las estrategias genéricas. Sobre la base del análisis de la industria, una compañía puede adoptar estrategias genéricas, que se denominan así porque pueden adecuarse a un amplio nivel para diferentes tipos de organizaciones; sin embargo, cualquier empresa puede usar más de una estrategia.

Según Richard L. Sandhusen, en su libro Mercadotecnia (citado por Thompson, 2016) proporciona la siguiente clasificación de estrategias para el crecimiento del mercado o estrategias de crecimiento para los productos ya existentes y para las nuevas adiciones al portafolio de productos:

Estrategias de Crecimiento Intensivo: Consisten en "cultivar" de manera intensiva los mercados actuales de la compañía. Son adecuadas en situaciones donde las oportunidades de "producto-mercado" existentes aún no han sido explotadas en su totalidad, e incluyen las siguientes estrategias.

Estrategia de penetración: Se enfoca en la mercadotecnia más agresiva de los productos ya existentes (por ejemplo, mediante una oferta de precio más conveniente que la de la competencia y actividades de publicidad, venta personal y promoción de ventas bastante agresiva). Este tipo de estrategia, por lo general, produce ingresos y utilidades porque 1) persuade a los clientes actuales a usar más del producto, 2) atrae a clientes de la competencia y 3) persuade a los clientes no decididos a transformarse en prospectos.

Estrategia de desarrollo de mercado: Se enfoca en atraer miembros a los nuevos mercados, por ejemplo, de aquellos segmentos a los que no se ha llegado aún (como nuevas zonas geográficas).

Estrategia de desarrollo del producto: Incluye desarrollar nuevos productos para atraer a miembros de los mercados ya existentes, por ejemplo, desarrollando una nueva presentación del producto que brinde beneficios adicionales a los clientes.

Por lo expuesto, penetrar un nuevo mercado demanda de una planificación exhaustiva que declaren los objetivos esperados, conocimiento del contexto a ingresar sea este nacional o internacional, la ley medioambiental, gustos, preferencias y hábitos de compra. Para autores como Becerra (2010); Mora et al. (2015); Bustamante y Feijóo (2015); el mercado consta de diversos tipos de clientes, productos y necesidades y el mercadólogo debe determinar cuáles segmentos ofrecen las mejores oportunidades. Los consumidores se pueden agrupar y atender de diversas maneras con base en factores geográficos, demográficos, psicográficos y conductuales. El proceso de dividir un mercado en distintos grupos de compradores con base a sus necesidades, características o comportamientos, podrían requerir de productos o mezclas de marketing disímiles.

Definir el posicionamiento competitivo de la compañía, alinear las actividades con la estrategia, construir una marcada diferencia con la competencia, asegurar una buena gestión de procesos, facilitar la sostenibilidad organizacional resultado de la actividad global de la compañía y no de las partes, así como de gestionar la eficiencia organizacional (Pulecio y Gómez, 2017). 


\section{Crecimiento Empresarial: Mipymes}

Algunos autores han planteado definiciones del término "crecimiento"; entre ellos Cardona y Cano (2005), expresan que el crecimiento es:

Proceso intangible que depende de elementos tangibles (acumulación de capital físico y humano), y sobre todo de una adecuada organización y estructura interna, el crecimiento se caracteriza por la acción compleja de las modificaciones internas que desembocan en un aumento de las dimensiones y cambios en las características de los objetos sometidos a tal proceso (p. 24) .

Cómo se percibe sobre todo en la primera definición de "crecimiento", para estos autores, el crecimiento es un proceso que se genera de adentro hacia afuera; la adecuada disposición de los recursos internos permite que la organización logre un posicionamiento externo que le permita crecer.

Por otro lado, Blázquez et al. (2006) manifiestan que el crecimiento es la expansión que realiza la empresa hacia nuevos mercados, tanto geográficos como de clientela, las compañías en crecimiento se expanden en nuevos mercados y segmentos de mercado, nuevas regiones, incluso nuevos países, el crecimiento permite que una empresa logre conseguir a los mejores empleados y los conserve y como es bien sabido, las capacidades, habilidades y competencias del personal se podrían constituir en fuente de ventaja competitiva.

Desde la perspectiva de Becerra (2010) el crecimiento empresarial es definido como:

El proceso de adaptación a los cambios exigidos por el entorno o promovidos por el espíritu emprendedor del directivo, según el cual, la empresa se ve compelida a desarrollar o ampliar su capacidad productiva mediante el ajuste o adquisición de nuevos recursos, realizando para ello cambios organizacionales que soporten las modificaciones realizadas; todo lo cual debe venir avalado por la capacidad financiera de la empresa que permita, a través de dicho proceso, obtener una competitividad perdurable en el tiempo (p. 16).

El crecimiento empresarial depende de diversos factores que pueden ser internos o externos. Entre los factores internos están la edad y el tamaño, la motivación, la estructura de propiedad y la gestión del conocimiento y entre los factores externos se pueden mencionar algunos relativos al entorno sectorial (competidores, clientes y proveedores), estos factores tienen diversas formas de medición; entre ellas se pueden citar el valor para los accionistas, las ganancias por acción, los beneficios y las ventas (Aguilera y Puerto, 2012).

Sin embargo, uno de los factores determinantes para lograr el crecimiento empresarial es el volumen de ventas; este factor es variable y depende en gran medida de la percepción, las preferencias, los gustos y los hábitos de compra de los clientes; recordar y relacionar la imagen de la empresa comprometida con la responsabilidad social arroja créditos ante el posicionamiento de imagen, la lealtad de los clientes y la identificación con la comunidad en la que opera la organización.

La mayoría de estudios realizados sobre el crecimiento empresarial tienden a centrarse en el éxito de las empresas grandes ya consolidadas más que en en el de los pequeños negocios, es decir 
que, existen pocos estudios previos que tratan sobre el crecimiento de las Mipymes y de los factores que influyen sobre las decisiones acerca de su crecimiento. Avolio (2018) señala que existen cuatro factores que influyen en el desempeño de las Mipymes: el desarrollo, la motivación por el crecimiento, las habilidades gerenciales para lograr dicho crecimiento y el acceso a los recursos y la demanda de mercado.

Las primeras Mipymes, surgieron como microempresas en la industria textil, el transporte y la industria metálica, seguido de la rápida expansión de las industrias del comercio y los servicios; sin embargo, a principios del siglo XX, las pequeñas y medianas empresas se convirtieron gradualmente en estructuras corporativas a gran escala (compañías y consorcios) capaces de transnacionalizar la gestión empresarial; multinacionales que nacieron del proceso de expansión del mercado (Jácome \& King, 2013). El paradigma empresarial capitalista se caracteriza por actividades económicas que pueden crear plusvalía, en las que el trabajo asalariado se considera una mercancía comprada a los trabajadores. Bajo esta lógica de gestión, la producción y los servicios han perdido su esencia social $y$ se han convertido en resultados económicos cada vez más concentrados, en un espacio donde la pequeña y mediana empresa suele perder competitividad en los mercados.

La composición empresarial de las Mipymes en Ecuador, se toman del Instituto Nacional de Estadísticas y Censo (INEC), así como de información proporcionada por el Servicio de Rentas Internas (SRI) y del Ministerio de Industrias y Productividad (MIP).
Según Baquerizo (2018), las Mipymes que actualmente existen en Ecuador, son las responsables del crecimiento de la producción, la generación de plazas de empleo, la innovación y el dinamismo productivo del país.

Previo al análisis respectivo del sector de la Mipyme, es imperativo puntualizar los parámetros de clasificación utilizados para definirla, de ahí se desprende que la clasificación utilizada es la establecida por la Comunidad Andina, con resolución N-1260, del 21 de agosto de 2009, la misma que es utilizada por el Instituto Nacional de Estadísticas y Censos (INEC), mencionado por Araque (2012), donde se señala que las empresas se clasifican según el número de trabajadores y el valor de sus ventas. Para la clasificación, en función del número de trabajadores, se domina qué microempresa emplea entre uno y nueve trabajadores. La pequeña empresa emplea entre 10 y 49 trabajadores; la mediana emplea entre 50 y 199 trabajadores, mientras que la gran empresa emplea a 200 o más trabajadores. La clasificación, en relación con el volumen de ventas, establece que la micro, pequeña, mediana y gran empresa, tienen ventas anuales de $\$ 1$ a $\$ 100.000$; de $\$ 100.001$ a $\$ 1.000 .000$; de $\$ 1.000 .001$ a $\$ 5.000 .000$ y desde $\$ 5.000 .001$, de forma respectiva.

En Ecuador estos tres sectores representan el 99,64\%, según cifras del INEC (2020). El segmento de mayor participación son las microempresas con un 91,35\%, seguido de las pequeñas con $6,75 \%$ y las medianas con $1,54 \%$. Las actividades económicas que concentran la mayor cantidad de Mipymes es comercio y servicio. Y las provincias con mayor Mipymes según Superintendencia de Compañías son: Guayas 37,85\%, Pichincha 36,75\%, Azuay 5,14\%, El oro 4,60\%, Manabí $3,96 \%$ y en otras provincias el $11,70 \%$. 
Figura 1

Participación de Mipymes por sector económico-Ecuador

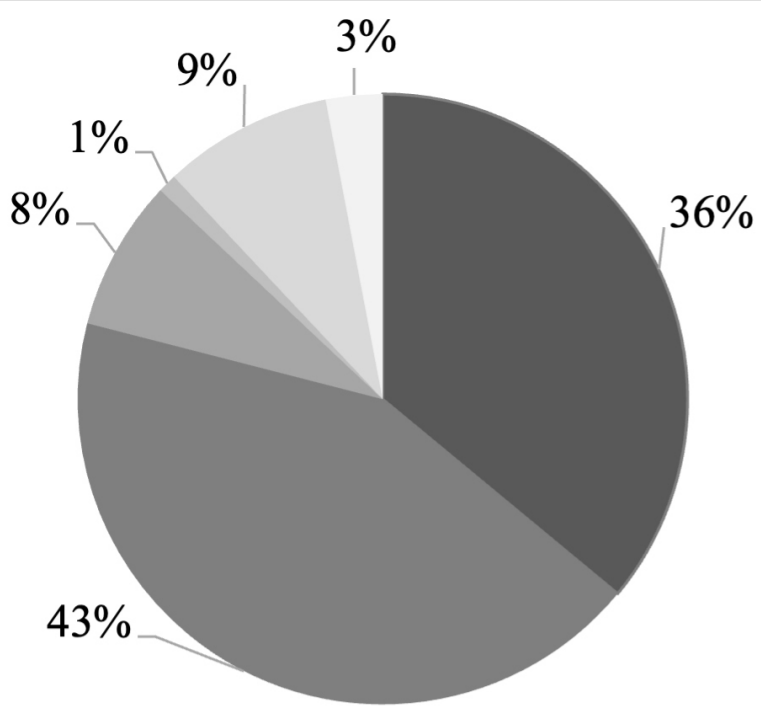

n Comercio

- Servicio

Industria

manufacturera

Explotacion de minas y cnateras

Agricultura, ganaderia, sivicultura y pesca

\section{Construccion}

Fuente: INEC 2019.

\section{METODOLOGÍA}

El presente estudio se enfoca en analizar las estrategias de desarrollo del mercado que han permitido que muchas Mipymes logren un crecimiento empresarial de forma general. Considerando el objetivo se planteó una investigación de tipo descriptiva bajo un enfoque cualitativo, no experimental, haciendo uso del método de investigación Desh Research, este implica el uso de datos ya existe. A través de una revisión bibliográfica minuciosa de artículos científicos, libros, folletos, artículos de prensa, datos institucionales, estado del arte, tesis, fuentes electrónicas situadas en la red web, entre otros, se recopilaron, analizaron y resumieron para incrementar la eficacia general de la investigación, y caracterizar de manera crítica la situación que presentan las Mipymes en Ecuador, pudiendo valorar cuáles han sido las estrategias que han desarrollado de manera particular algunos negocios ecuatorianos para alcanzar su crecimiento, sobrevivir y desarrollarse en el entorno económico del país y llegar a conclusiones críticas precisas sobre el tema estudiado.

\section{RESULTADOS}

Ecuador es un país de Mipymes en donde juegan un rol importante en el desarrollo económico del país, estas aportan empleo y riqueza y son el 99,64\% del tejido empresarial. Sin embargo, existen algunos factores que las Mipymes deben tener en cuenta al aplicar las diferentes estrategias de desarrollo del mercado las cuales se definen como el conjunto de acciones por medio de las cuales una organización espera 
satisfacer las necesidades de su mercado objetivo, el diseño de estrategias puede llevarse a cabo a nivel de posicionamiento, mezcla, calidad del

Figura 2

Distribución de Mipymes por tamaño

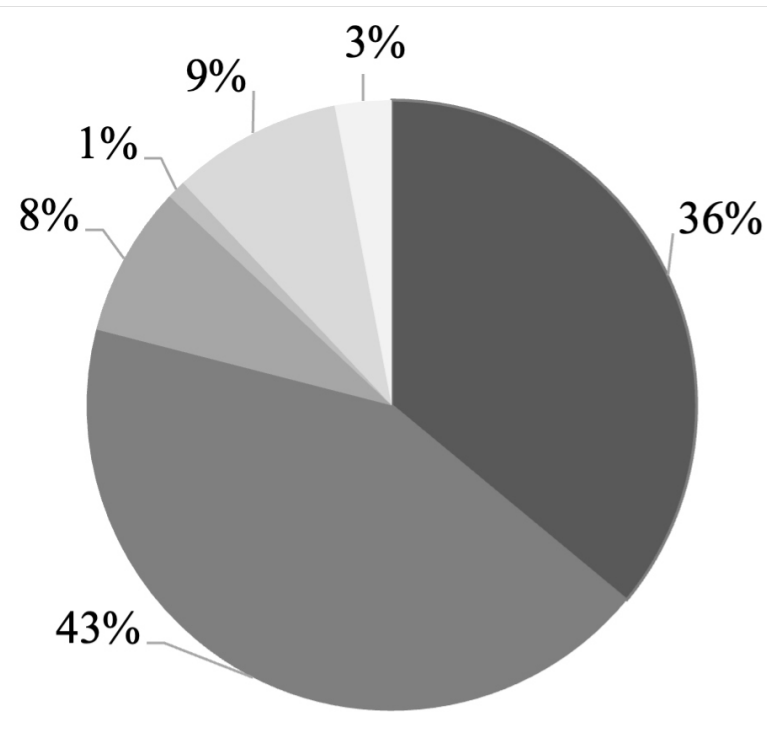

servicio, satisfacción del cliente, gastos de mercadeo y creación de valor como ventaja competitiva.
Industria manufacturera

\section{Explotacion de minas y cnateras}

\section{Agricultura, ganaderia, sivicultura y pesca}

\section{Construccion}

Fuente: INEC 2019.

En Ecuador, de cada 100 unidades empresariales, aproximadamente 99 están clasificadas como Mipymes y emplean a un 75\% del total de la masa laboral, las cuales hoy en día aplican nuevas estrategias empresariales. El 91,35\% de unidades económicas esta representado por el sector microempresarial, el $6,75 \%$ por las pequeñas y un $1,54 \%$ por medianas empresas.

Algunas dificultades exigen a las Mipymes en Ecuador emplear nuevas estrategias de desarrollo ante este entorno sistémico, complejo e incierto; pero sobre todo se ha hecho evidente la necesidad de actuar reactivamente, para afrontar los retos impuestos por la pandemia de la Covid-19, así como sus implicaciones sanitarias, sociales $y$ económicas. Para tal efecto se muestra en la siguiente tabla algunos ejemplos de Mipymes ecuatorianas que para alcanzar crecimientos y sosteniblidad han aplicado algunas estrategias de desarrollo empresarial.

Entre los negocios ecuatorianos que han implementado estrategias de desarrollo de mercado tenemos: La holandesa, Alpina, El ordeño, Cerveceria Nacional, Tonicorp y Salas \& Hahl. 
Tabla 1

Estrategia de desarrollo de mercado en Mipymes Ecuatorianas.

\begin{tabular}{lc}
\hline Estrategias & Porcentajes \\
\hline Desarrollo del mercado diferenciado & $66,70 \%$ \\
Modificación de los productos existentes & $74,10 \%$ \\
Estrategia de precios & $25,90 \%$ \\
Distribución de mercadeo directo al consumidor & $55,60 \%$ \\
Promoción de productos & $63,00 \%$ \\
\hline
\end{tabular}

Fuente: Diario El Universo.

La tabla describe algunas de las principales estrategias de desarrollo de mercado que utilizan las Mipymes ecuatorianas. Estos agentes económicos que caracterizan por ser resilientes ante las situaciones de las crisis económicas. Tal es el caso que, ante la pandemia por Covid-19, la mayoría no quedó rezagada, sino más bien aprovechó la situación para reinventarse, viéndola como una oportunidad. Aplicaron estrategia de desarrollo de mercado entre las que se detalla: estrategia de desarrollo del mercado diferenciado con el $(66,7 \%)$. Entre las estrategias de la mezcla de mercadeo se destaca un 74,1\% con la modificación de los productos existentes, en tanto que, con la estrategia de precios está orientada la competencia (25,9\%). Para su distribución se emplea con un $55,6 \%$ el mercadeo directo al consumidor, mientras que en la promoción resalta la comunicación masiva con un 63,0\%.

La innovación fue y es clave para poder subsistir. Muchas innovaron y lanzaron nuevos productos al mercado, algunos proyectados desde antes de la pandemia y otros que nacieron a raíz de ella, como el caso de la empresa La holandesa y Salas \& Hahl, aplicaron nuevas estrategias empresariales, que antes de la pandemia se dedicaban a importar productos varios y por el desplome de sus ingresos idearon en abril pasado nuevos productos como el de un llavero que impida tener contacto con superficies de alto tráfico y la buena acogida hizo que la producción se incremente gracias a que lograron codificarse con Megamaxi y estar presentes a nivel nacional con 14 puntos de venta.

Por otro lado, en negocio El ordeño, con su marca de leches saborizadas TRÜ, incorporaron tres nuevos sabores y el primer sorbete de papel en empaque tetra pak. Antes de la pandemia la gerente de marketing de la empresa ya tenía planificado el lanzamiento de gran parte del portafolio de TRÜ, sin embargo, a raíz de la pandemia se hizo algunos ajustes tomando en cuenta los cambios que existen en el mercado y en el consumidor.

Las Mipymes ecuatorianas para desarrollar sus mercados tuvieron que recurrir a la reinvención organizacional desde los Objetivos de Desarrollo Sostenible, reconversión del marketing tradicional al marketing digital, resiliencia, y resguardar la salud en base para generar ingresos y que sus 
productos sean aceptados en el mercado.

El fuerte impacto que acarreó la Covid-19 a la economía ecuatoriana, ha creado en la Mipymes la necesidad urgente de reinventarse. La crisis ha cambiado la forma en que funcionan estas empresas y ha obligado a pensar a este grupo de agentes económicos. Se registraron 281 empresas en el país que abrieron una nota más a su RUC para crear, por ejemplo, mascarillas y otros insumos médicos.

Ese es el caso de 48 Mipymes en Ecuador que se reinventaron $\mathrm{y}$ ofrecieron por primera vez un producto o llegaron a un nuevo mercado, empezaron con pocos productos y han ampliado y diversificado las ofertas, la mayoría se concentró en las provincias más pobladas como Pichincha, Guayas, Manabí y Azuay.

Las tiendas repletas de productos de primera necesidad son un nuevo nicho de mercado. Decenas de emprendedores adecuaron y crearon estos locales, propietarios de bares y discotecas, hoteleros, entre otros, instalaron minimarkets en sus casas o en los locales de sus anteriores negocios. Se crearon muchos nuevos negocios de acuerdo con la necesidad del momento por la pandemia por la Covid-19.

\section{DISCUSION}

Definir el medio sobre el cual va a operar una empresa y posicionarse mejor en el mercado es el desafío que tiene el sector de las Mipymes. Una estrategia es un plan general de acción, mediante el cual una organización busca alcanzar sus objetivos (Walker et al. 2007). Las estrategias, permiten concretar y ejecutar los proyectos estratégicos, pues responden a la interrogante sobre cómo lograr y hacer realidad cada objetivo siempre enfocado en las necesidades y deseos del cliente y las oportunidades que dada la situación del entorno se identifiquen.

Las diferentes vías tanto para el crecimiento empresarial como de aplicación de estrategias de desarrollo del mercado están en función de lo que mencionan Aguilera y Puerto (2012). Este depende de diversos factores que pueden ser tanto internos como externos y que tienen diversas formas de medición, entre ellas se puede citar el valor para los accionistas, las ganancias por acción, los beneficios y las ventas esperadas por la organización, así como del momento socioeconómico que viva cada contexto.

Las estrategias, están orientadas a las variables que la empresa puede controlar y que responden a las exigencias de comercialización de sus productos. La estrategia de desarrollo del mercado debe especificar los segmentos de mercado a los que se dirigirá la empresa, es decir, debe concentrarse en los clientes que puede satisfacer mejor en todo momento cubriendo el sector de servicio y comercio que según el INEC (2017) son los más apetecidos por las Mipymes en el mercado ecuatoriano.

Si bien el año 2020 ha sido un año difícil, por la crisis ante la pandemia de la Covid-19, hay quienes han encontrado oportunidades, es el caso de las Mipymes (Chavarro, 2020). Entre las estrategias de desarrollo de mercadeo se destaca la modificación de los productos existentes, en tanto que, la estrategia de precios está orientada a la competencia. Para su distribución se emplea el mercadeo directo al consumidor, mientras que en la promoción resalta la comunicación masiva. Esta estrategia de diferenciación consiste en hacer que 
una empresa que ofrezca un producto en el mercado y teniendo rivales compitiendo en el sector, sea capaz de destacar de forma significativa. Esto gracias a cualidades, características, tecnologías y otros factores que la hacen positivamente diferente a las demás marcas que ofrecen productos o servicios similares. A esto se le denomina creación de valor o ventaja competitiva.

En lo referente a la mezcla de productos, es la variedad de artículos ofrecidos por una empresa al público. Entre sus ventajas está la posibilidad de fidelizar a los clientes y garantizar una mayor cuota del mercado. Asegurar una estrategia efectiva a este respecto es esencial para la salud de cualquier emprendimiento simplemente porque el demandante siempre quiere encontrarlo todo en un solo lugar.

Manteniendo la línea de pensamiento de Smith (1776); Kotler (1998); Walker et. al. (2007); Cancino et al. (2012) y Adame et al. (2017), la actividad emprendedora es fundamental para el desarrollo económico de una Mipymes, este vínculo se observa cuando los emprendedores inventan nuevos negocios, los nuevos negocios necesitan producir empleos, se intensifica la competencia e inclusive se incrementa la productividad por medio de cambios tecnológicos. Esto se ha observado actualmente en el mundo, de manera especial en Ecuador debido a la pandemia que por Covid-19 ha enfrentado el sector empresarial desde marzo del año 2020, teniendo que intensificar la creatividad, planear nuevos satisfactores para los clientes, trabajar mediante la modalidad de teletrabajo, llegar al segmento o nicho de mercado vía delivery, vender a través del e-commerce e intercambiar productos haciendo uso de la tecnología vitalmente en pleno siglo XXI. En una época en donde salir de la casa se convertía en un riesgo para la población.

Una parte fundamental para formular una estrategia de desarrollo del mercado es determinar con exactitud a cuáles segmentos del mercado a las Mipymes dirigirán sus esfuerzos; dichos segmentos se diferencian según las necesidades, deseos y oportunidades que brinde el mercado y, las diferentes respuestas al mercadeo y sus utilidades. Las Mipymes exitosas reconocerán la importancia de orientar todos sus esfuerzos hacia aquellos segmentos del mercado que pueda satisfacer mejor desde un punto de vista competitivo, desarrollando luego, una estrategia para cada mercado meta, nichos de mercados y sectores productivos. Todo depende de conocer lo que desea el cliente, solo así la empresa emprende la estrategia que logrará solucionar esa insatisfacción.

\section{CONCLUSIONES}

Actualmente, el sector de las Mipymes se encuentra en medio de condiciones adversas, caracterizadas por la sobreoferta de productos y servicios y altos niveles de incertidumbre. Por ser Mipymes se lucha por tener mejor posicionamiento en el mercado, lo cual también es producto de las estrategias aplicadas internamente. El principal desafío que enfrentan las Mipymes es la globalización de los mercados, que ejerce presión hacia la eficiencia y obliga a todos los agentes participantes a incrementar su competitividad para, por lo menos, permanecer en el mercado, cada vez más volátil y exigente, jugando un papel importante la flexibilidad operacional y las estrategias de mercado, lo que 
permite e invita a la innovación continua.

Mientras tanto, la vinculación de la estrategia de desarrollo del mercado con una planificación estratégica auténtica debe evaluar la realidad del mercado, las oportunidades que este presente y su relación con las capacidades internas, las empresas con una orientación hacia el crecimiento empresarial deben basarse en una visión externa de los clientes y competidores, lo cual se traduce en un conjunto de actitudes y un sistema de valores que se infiltre en todas las funciones del negocio. Operar bajo este concepto requiere de un conocimiento y un compromiso profundo por parte de toda la empresa, y no sólo del departamento que lo administra. La resiliencia en el sector de las Mipymes, marca puntos a su favor para poder seguir operando en un mercado en donde los retos son cada vez mayores.

Todo esto nos lleva a aclarar que el sector de las Mipymes debe estar en constante cambio, abriendo nuevas posibilidades de asentamiento en el mercado y con esto expandir el desarrollo económico empresarial, especializándose en lo que mejor se sabe hacer y con la división del trabajo y apoyo de marketing como la herramienta posibilitadora de posicionamiento de marca, llevar al consumidor lo que busca por necesidad o deseo en algún momento del tiempo. Como se puede inferir, en estos negocios, el dueño, tiene la cualidad de ser al mismo tiempo administrador de su propia empresa. Lo importante aquí es que las Mipymes deben ser resilientes, sus dueños capacitarse en definiciones que le permitan un mejor posicionamiento porque con su actividad contribuyen al desarrollo local, a la creación de empleos y al aumento del ingreso familiar, donde se aprovechan los recursos internos que existen en las zonas rurales centro urbano de cualquier país en vías de desarrollo o desarrollado. 
$\begin{array}{lllllll}\text { R } & \text { E } & \text { V } & \text { I } & \text { S } & \text { T } & \text { A }\end{array}$

Public a n d o

I S S N $133900-9304$

\section{REFERENCIAS}

Adame, L. C. G., Arreola, R. J. y Paniagua, M. L. O. (2017). Crecimiento empresarial \& Mipymes de Autlán de Navarro. Revista Cubana de Contabilidad y Finanzas. COFIN HABANA, (1), 131-142. http:// www.cofinhab.uh.cu/index.php/RCCF/ article/view/215

Aguilera, A., y Puerto, P. (2012). Crecimiento empresarial basado en la Responsabilidad Social. Pensamiento \& gestión, (32), 1-26. h t t p : / / w w w. s ciel o. or g.co / scielo.php?pid=S165762762012000100002 \&script=sci_arttext\&tlng=en $\quad$ http:// w w w. s c i e 1 o.org.col scielo.php?script $=$ sci_arttext\&pid $=\$ 1657-6$ 2762012000100002

Araque, W. (2012). Situación actual y proyección del sector productivo con énfasis en las Mipymey el emprendimiento. Observatorio de la pyme. UASB. Recuperado a partir de https:/ /slideplayer.es/slide/3227755/

Avolio, B. (2018). Factores que Limitan las micro y pequeñas empresas en el Perú (MYPES). Srategia, 71-72. https://www.academia.edu/ $\begin{array}{lllllllll}3 & 2 & 8 & 4 & 7 & 5 & 9 & 2 & 1\end{array}$ Factores_que_limitan_el_crecimiento_de_1 as_micro_y_peque $\% \mathrm{C} 3 \% \mathrm{~B} 1$ as_empresas_e n_el_Per\%C3\%BA_MYPES

Baquerizo (2018). Mipymes y Organizaciones de Economía Popular y Solidaria son una pieza clave para la economía del país. Servicio Ecuatoriano de Normalización INEN. https://www.normalizacion.gob.ec/ mipymes-y-organizaciones-de-economia- popular-y-solidaria-son-una-pieza-clavepara-la-economia-del-pais/

Becerra, D. P. P. (2010). La globalización y el crecimiento empresarial a través de estrategias de internacionalización. Pensamiento \& gestión, (28), 171-195. https:// www.redalyc.org/pdf/646/64615176009.pdf

Blázquez Santana, F., Dorta Velázquez, J. A., \& Verona Martel, M. C. (2006). Concepto, perspectivas y medida del crecimiento empresarial. Cuadernos de administración, 19(31), 165-195. Obtenido de http:// www.scielo.org.co/pdf/cadm/v19n31/ v19n31a07.pdf?iframe=true\&width

Bustamante, B. F., y Feijóo, A. E. (2015). Formulación de estrategias de crecimiento empresarial para imprenta Gráficas Feijóo (tesis de grado, Universidad Politécnica Salesiana). https://dspace.ups.edu.ec/handle/ $123456789 / 7838$

Cancino, C. A., Coronado, F., y Farias, A. (2012). Antecedentes y resultados de emprendimientos dinámicos en Chile: cinco casos de éxito. INNOVAR. Revista de Ciencias Administrativas y Sociales, 22(43), 19-32. https://www.redalyc.org/pdf/ 818/81824123003.pdf

Cardona, C. A., y Cano, C. A. (2005). Territorio, ciclo de vida y estructura empresarial: un puente en la industrialización regional. Universidad Eafit.

Ceupe. (21 de Septiembre de 2016). Las estrategias de Crecimiento empresarial. CEUPE magazine. https://www.ceupe.com/ 
blog/las-estrategias-de-crecimientoempresarial.html

Chavarro, S. (7 de Junio de 2020). La emergencia sanitaria en Ecuador sirvió para emprender y reacondicionar. El comercio. https:// www.elcomercio.com/actualidad/ emergencia-sanitaria-ecuadoremprendimientos-negocios.html

Donawa, M. C. (2018). Fuerzas competitivas que moldean la estrategia en la gerencia del sector mipymes del distrito de Santa MartaMagdalena. Obtenido de Revista EAN: https://doi. org/ 10.21158/01208160.n84.2018.1919

INEC. (2019). Mipymes y Organizaciones de Economía Popular y Solidaria son una pieza clave para la economía del país. https:// www.normalizacion.gob.ec/mipymes-yorganizaciones-de-economia-popular-ysolidaria-son-una-pieza-clave-para-laeconomia-del-pais/

Jácome, H., y King, K. (2013). Estudios Industriales de la micro, pequeña y mediana empresa. Quito: $\quad$ FLACSO. https:// biblio.flacsoandes.edu.ec/libros/digital/ 52089.pdf

Kotler, P. (1998). Estrategias de mercadeo de las Mipymes. Redalyc, 32.

Macías, L. A. (2015). Análisis sectorial de las micro, pequeñas y medianas empresas (MIPYMES) de la provincia del Guayas, período 2011-2013 (Tesis de maestría, Universidad de Guayaquil). http:// repositorio.ug.edu.ec/handle/redug/8956

Mora, E. H., Vera, M. A., y Melgarejo, Z. A. (2015).
Planificación estratégica y niveles de competitividad de las Mipymes del sector comercio en Bogotá. Estudios gerenciales, 31(134), 79-87. https:// www.sciencedirect.com/science/article/pii/ $\underline{\text { S0123592314001600 }}$

Parrales Poveda, M., Gracia Panta, E., Miller Zavala, J., \& Ponce Cedeño, O. (2017). Lineamientos Estratégicos y Comunicación Digital (Primera ed.). Mawil Publicaciones de Ecuador.

Pulecio, C., y Gómez, H. (2017). Planeación estratégica para MIPYMES. https:// repository.ucc.edu.co/handle/ 20.500.12494/20085

Riquelme, M. (31 de Julio de 2015). Estrategia de Desarrollo de Mercado. Web y empresas. https://www.webyempresas.com/estrategiade-desarrollo-de-mercado/

Smith, A. (1776). Investigación de la naturaleza y causas de la riqueza de las naciones.

Thompson, I. (2 de Abril de 2016). Estrategias de mercado. Promo negocios. https:// www.promonegocios.net/mercado/ estrategias-mercado.html

Walker, S. E., Etzel, M., y Stanton, W. (2007). Fundamentos de marketing. Editorial McGraw Hill. https:// mercadeo1marthasandino.files.wordpress.c om/2015/02/fundamentos-de-marketingstanton-14edi.pdf 